\title{
Paediatric multisystem inflammatory syndrome associated with COVID-19: filling the gap between myocarditis and Kawasaki?
}

\author{
Jeanne Bordet ${ }^{1}$ - Stéphanie Perrier ${ }^{2}$ - Catherine Olexa ${ }^{1,2} \cdot$ Anne-Cécile Gerout $^{3}$ - Philippe Billaud ${ }^{2}$. \\ Laurent Bonnemains ${ }^{1,2,4} \mathbb{B}$
}

Received: 13 July 2020 / Revised: 28 August 2020 / Accepted: 10 September 2020 / Published online: 21 September 2020

(C) Springer-Verlag GmbH Germany, part of Springer Nature 2020

\begin{abstract}
Myocarditis and Kawasaki disease are common but usually distinct diseases in children. During the coronavirus pandemic (COVID-19), reports of a new form of myocarditis with clinical features of Kawasaki appeared. We investigated the place of this new disease in the spectrum encompassing Kawasaki disease and myocarditis.

Thirty two consecutive children referred to our centre for a suspicion of Kawasaki or a diagnosis of myocarditis were included and eventually divided into four groups: 11 Kawasaki diseases, 6 Kawasaki syndromes (children with another diagnosis), 7 myocarditis without Kawasaki clinical feature and 7 myocarditis with incomplete Kawasaki clinical features. All were treated with immunoglobulins except those of the myocarditis group. The survival rate was $91 \%$. The 7 children with myocarditis and clinical features of incomplete Kawasaki were all positive for SARS-CoV-2. They had a transient myocardial failure with a favourable course and none had coronary artery disease.

Conclusion: Every COVID-19 child within our population had a mild to severe myocarditis and presented with fever plus two or three Kawasaki clinical features. Short-term evolution was good for these children. This new disease seems to fill the gap between isolated myocarditis and Kawasaki disease.
\end{abstract}

What is Known:

- A new paediatric disease close to Kawasaki disease appeared during the COVID-19 pandemic

What is New:

- In our population, children presented with fever, vivid Kawasaki clinical features (although the Kawasaki syndrome was always incomplete) and a myocarditis without coronary abnormalities.

- The new disease fills the gap between paediatric myocarditis and Kawasaki disease but its prognosis is much better.

Keywords Kawasaki disease $\cdot$ Myocarditis $\cdot$ Paediatric vascularitis $\cdot$ COVID-19 $\cdot$ SARS-COV-2

Laurent Bonnemains

laurent.bonnemains@inserm.fr

Jeanne Bordet

jeanne.bordet@chru-strasbourg.fr

Stéphanie Perrier

stephanie.perrier@chru-strasbourg.fr

Catherine Olexa

Catherine.olexa@chru-strasbourg.fr

Anne-Cécile Gerout

anne-cecile.gerout@chru-strasbourg.fr
Philippe Billaud

philippe.billaud@chru-strasbourg.fr

1 Cardiologie infantile, CHU Hautepierre, Strasbourg University Hospital, 1 rue de Molière, 67200 Strasbourg, France

2 Cardiac Surgery, New Civil Hospital, Strasbourg University Hospital, rue de l'Hopital, 67000 Strasbourg, France

3 Pharmacy, CHU Hautepierre, Strasbourg University Hospital, 1 rue de Molière, 67200 Strasbourg, France

4 iCube - UMR 7357, 2 rue Boussingault, 67000 Strasbourg, France 


\section{Abbreviations \\ COVID-19 Coronavirus disease 2019 \\ CT Computed tomography \\ ICD-10 10th edition of the International \\ Classification of Disease \\ PCR Polymerase chain reaction \\ SARS-CoV-2 Severe acute respiratory syndrome \\ due to coronavirus 2}

Kawasaki disease is an acute vasculitis affecting mostly infants and children with an increased risk of coronary artery aneurism formation [1,2]. Diagnosis is primarily based on a precise epidemiological case definition [3]: fever persisting at least 5 days and presence of at least 4 principal clinical features amongst acute or subacute changes in extremities, polymorphous exanthema, bilateral bulbar conjunctival injection without exudate, changes in lips and oral cavity, cervical lymphadenopathy and exclusion of other diseases with similar findings. Also, patients with at least 5 days of fever and less than 4 of the principal criteria can be diagnosed with Kawasaki disease when coronary artery dilation is detected by echocardiography or angiography [3]. Diagnostic criteria have been notably revised in 2004 with the conceptual introduction of incomplete Kawasaki disease for children with unexplained fever during 5 days or more and presenting with 2 or 3 of the principal clinical features of Kawasaki disease and at least four of seven supplemental laboratory criteria [3]. With the onset of the coronavirus pandemic (COVID-19) in Europe at the beginning of 2020, many reports have described cases of severe paediatric multisystem inflammatory syndrome mimicking incomplete Kawasaki disease [4]. These children usually presented with myocarditis but had very little coronary involvement. They were older children who had been infected with the new severe acute respiratory syndrome-coronavirus 2 (SARS-CoV-2). Our centre is located in eastern France, an area particularly impacted with SARS-CoV-2 pandemic [5]. The aim of our study was to better describe and to position this new disease within the spectrum encompassing Kawasaki diseases and myocarditis.

\section{Methods}

\section{Patients}

This retrospective study included every consecutive child (age $\leq 18$ years) who presented at our centre between January 2018 and May 2020 with one of the following criteria: (1) patient treated with intravenous human immunoglobulin for Kawasaki syndrome. In our country, the delivery of human immunoglobulin implies a declaration at the hospital pharmacy. This pharmaceutical record was screened for the word "Kawasaki". The indication for immunoglobulins was verified in the patient clinical file which contained the expressions "Kawasaki" or "suspicion of Kawasaki". (2) Patient with a final diagnosis of myocarditis i.e. an ICD-10 hospital discharge code in the list $\{\mathrm{I} 40, \mathrm{I} 41\}$ (the 10th revision of the international classification of diseases ICD10 has been used in our hospital during the whole duration of the study).

Thirty-six patients were identified and each medical record was then analysed. Two patients were also discarded because the real diagnosis according to the medical record was different (ICD coding errors). Two patients who had been included because they had received immunoglobulins for a suspected Kawasaki syndrome, were eventually excluded because the medical report did not meet the clinical or ultrasound criteria for even incomplete Kawasaki syndrome. Patients were divided in 4 groups according to their respective diagnoses:

1 Kawasaki disease (KD group), complete or incomplete, according to the usual definition [3]: patients either with a complete Kawasaki form, or with echocardiographic coronary dilatation, or with more than two clinical criteria and $\mathrm{CRP}>30 \mathrm{mg} / \mathrm{L}$ and at least three supplemental laboratory criteria

2 Kawasaki syndrome (KS group): patient complying with the clinical definition of the Kawasaki disease but eventually having another diagnosis (such as viral infection or toxic shock syndrome)

3 Isolated myocarditis (MY group): children with a myocarditis and no more than two of the five Kawasaki clinical features

4 All the other patients (KM group).

\section{Diagnoses}

The diagnoses of Kawasaki in this study were based on the American Academy of Pediatrics definition [3]. Myocarditis was clinically suspected on the basis of the combination of elevated serum levels of troponin and echocardiographic finding of ventricular dysfunction [6]. Diagnosis of COVID-19 was based on contagion history, nasal and/or stool polymerase-chain-reaction testing, and/or SARS-CoV-2 serology, and/or computational tomography (CT scan) [7]. Serological diagnostic tests had only been available in our centre since April 2020. All the patients of the cohort hospitalized after the 14th of March 2020 were at tested for SARSCoV-2 infection.

\section{Data and outcomes}

Patients' data were anonymized, in compliance with our ethics committee recommendations. Clinical, biological, electrocardiographic, echocardiographic and magnetic-resonance 
imaging data were collected. Two outcomes were analysed: mortality defined as death occurring during the same hospitalization or within 30 days after the admission and the aftereffect of the disease defined as persistent myocardial dysfunction at hospital discharge or coronary dilatation abnormality.

\section{Statistics}

Data were expressed as median [minimum value; maximum value]. Statistical analysis was performed using R 3.6.0 (R Foundation for Statistical Computing, Vienna, Austria) [8]. Continuous variables were compared by non-parametric Mann-Whitney $U$ test adapted for small groups (directly coded within the wilcox.test $\mathrm{R}$ function) [9]. Two-categorical variables were analysed by Fisher exact test. A value of $p<0.05$ was considered of statistical significance. Variables with more than $15 \%$ of missing data were excluded from the analysis.

\section{Results}

Study flow chart is presented in Fig. 1. Main characteristics of the study population are given in Table 1. Clinical signs are described for each diagnosis in Table 2. In the KD group, no child had an incomplete Kawasaki form, two had been diagnosed because of the echocardiographic findings and 9 presented a complete Kawasaki form. In the KD, KS or MY groups, no child had a COVID-19 diagnosis, whereas the seven children of group KM were COVID positive: 1 case was confirmed by nasal PCR, 1 case diagnosed in front of typical bilateral thoracic CT scan images, 3 had positive

Fig. 1 Flowchart of the study plasma serology and 2 cases had both typical CT images and positive serology.

\section{Patients with COVID+ myocarditis}

Amongst those patients, 1 had asthma and another had macrocephaly with moderate psychomotor retardation. In this subgroup, fever was the first symptom in all cases. Abdominal pain was often present (57\%, 4 cases on 7). Other initial clinical signs were cervical swelling, skin rash or diarrhoea. We also reported 2 cases of chest pain (29\%), 3 cases of shock syndrome (43\%), 3 cases of dyspnoea (43\%) and 3 cases of neurological disorders: visual hallucination, ideation slowing down or febrile meningeal syndrome ( 2 cases, $29 \%$ ). In many cases, symptoms of Kawasaki disease were predominant in the clinical picture (Fig. 2). Biological inflammatory syndrome was noted in all cases. C-reactive protein plasmatic levels in this subgroup were higher than in the rest of the study population: 248 [48-354] mg/L vs 135 [25-416] mg/L, respectively $(p=0.04)$. Five patients $(71 \%)$ were tested positive for lupus anticoagulant. Amongst those, one patient was secondarily tested negative, favouring the transitory status of positive lupus anticoagulant. None of these patients had history of systemic lupus erythematosus. There was one case (14\%) of macrophages activation syndrome confirmed after bone marrow analysis. Echocardiography on admission found an altered LV contractility in all cases, with a reduced left ventricle ejection fraction in 6 cases (86\%). Isolated reduction of longitudinal global strain was objectified in one case. Mitral regurgitation was present in all cases without annular dilatation. All patients were free from coronary involvement. Amongst the 7 patients diagnosed with incomplete

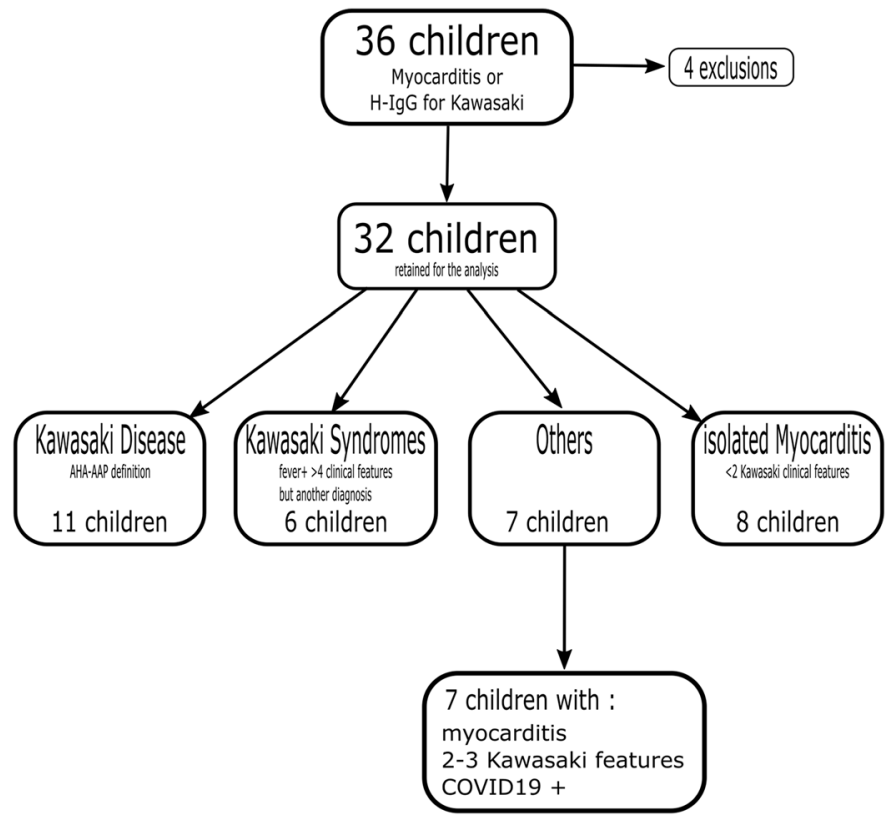


Table 1 Characteristics of the study population

\begin{tabular}{|c|c|c|c|c|}
\hline Variables & Total $(n=32)$ & $\begin{array}{l}\text { Before pandemic } \\
(n=21)\end{array}$ & $\begin{array}{l}\text { During pandemic } \\
(n=11)\end{array}$ & $p$ \\
\hline Female & $15(43.8 \%)$ & $10(47.6 \%)$ & $4(36.4 \%)$ & 0.712 \\
\hline Age (years) & $5[0.1-18]$ & $5[0.6-17]$ & $7[0.1-15]$ & 0.046 \\
\hline Weight (kg) & $20.8[4.7-75]$ & $21.5[6-60]$ & $34.1[4.7-60]$ & 0.061 \\
\hline Height (cm) & $1.1[0.5-1.8]$ & $103[68-180]$ & $127[54-174]$ & 0.034 \\
\hline Duration of fever (days) & $5[0-48]$ & $3[0-48]$ & $4[2-5]$ & 0.755 \\
\hline \multicolumn{5}{|l|}{ Diagnosis } \\
\hline Kawasaki disease (KD) & $11(34.4 \%)$ & $9(42.9 \%)$ & $2(18.2 \%)$ & 0.248 \\
\hline Kawasaki syndrome (KS) & $6(18.7 \%)$ & $6(28.6 \%)$ & 0 & 0.071 \\
\hline $\begin{array}{l}\text { Isolated myocarditis } \\
\text { (MY) }\end{array}$ & $8(25 \%)$ & $6(28.6 \%)$ & $2(18.2 \%)$ & 0.681 \\
\hline Other (KM) & $7(21.9 \%)$ & 0 & $7(63.6 \%)$ & $<0.0001$ \\
\hline \multicolumn{5}{|c|}{ Biology (peak levels during hospitalization) } \\
\hline C-reactive protein $(\mathrm{mg} / \mathrm{L})$ & $\begin{array}{l}154.8 \\
\quad[25-416.2]\end{array}$ & $50.7[25-404]$ & $258[30-354]$ & 0.381 \\
\hline White cells (G/L) & $13.3[1.4-53]$ & $9.5[3.9-28.5]$ & $10[7.9-20.1]$ & 0.846 \\
\hline Neutrophils (G/L) & $8.3[0.4-51.4]$ & $6.1[2.6-19.6]$ & $8.3[1.8-16.4]$ & 0.636 \\
\hline \multicolumn{5}{|c|}{ Electrocardiogram on admission } \\
\hline Abnormal repolarization & $5(16.7 \%)$ & $4(21.1 \%)$ & $1(9.1 \%)$ & 0.626 \\
\hline \multicolumn{5}{|l|}{ Echocardiography findings } \\
\hline $\operatorname{LVEF}(\%)$ & $54.8[10-70]$ & $60[30-70]$ & $60[10-65]$ & 0.157 \\
\hline LV dysfunction & $13(41.9 \%)$ & $6(30 \%)$ & $7(63.6 \%)$ & 0.128 \\
\hline RV dysfunction & $2(6.5 \%)$ & $2(10 \%)$ & 0 & 0.527 \\
\hline Mitral valve regurgitation & $16(51.6 \%)$ & $7(35 \%)$ & $9(81.8 \%)$ & 0.023 \\
\hline Pericardial effusion & $12(38.7 \%)$ & $7(35 \%)$ & $5(45.5 \%)$ & 0.705 \\
\hline $\begin{array}{l}\text { Coronary } \\
\text { dilatation/aneurysm }\end{array}$ & $5(16.1 \%)$ & $4(20 \%)$ & $1(9.1 \%)$ & 0.631 \\
\hline \multicolumn{5}{|l|}{ Treatment } \\
\hline Mechanical ventilation & $5(15.6 \%)$ & $4(19 \%)$ & $1(9.1 \%)$ & 0.637 \\
\hline Inotropes & $9(28.1 \%)$ & $4(19 \%)$ & $5(45.5 \%)$ & 0.213 \\
\hline Immunoglobulins & $24(75 \%)$ & $15(71.4 \%)$ & $9(81.8 \%)$ & 0.681 \\
\hline Steroids & $14(43.8 \%)$ & $8(38.1 \%)$ & $6(54.5 \%)$ & 0.465 \\
\hline Aspirin & $23(71.9 \%)$ & $16(76.2 \%)$ & $7(63.6 \%)$ & 0.681 \\
\hline Antibiotics & $2681.3 \%)$ & $16(76.2 \%)$ & $10(90.9 \%)$ & 0.637 \\
\hline Heparin & $11(34.4 \%)$ & $5(23.8 \%)$ & $6(54.5 \%)$ & 0.123 \\
\hline \multicolumn{5}{|l|}{ Outcomes } \\
\hline Hospital stay (days) & $7[0-40]$ & $5[2-40]$ & $8[5-17]$ & 0.233 \\
\hline ICU stay (days) & $0.6[0-19]$ & $2[0-19]$ & $4[0-10]$ & 0.104 \\
\hline Incomplete recovery & $4(12.9 \%)$ & $3(15 \%)$ & $1(9.1 \%)$ & 1 \\
\hline Death & $3(9.4 \%)$ & $3(14.3 \%)$ & 0 & 0.534 \\
\hline
\end{tabular}

$I C U$ intensive care unit, $L V$ left ventricle, $L V E F$ left ventricular ejection fraction, $R V$ right ventricle
Kawasaki disease associated with myocarditis, 5 had cardiac resonance imaging performed within 10 days. In all cases, cardiac resonance imaging was normal, without myocardial oedema. One patient, from this group, initially presented with a clinical picture of acute abdomen leading to laparoscopic appendectomy. Evolution was marked by persistent fever and severe inflammatory syndrome. The patient secondarily developed signs of cardiac failure requiring inotropic support, prolonged ventilation during 5 days. Cardiac function improved after infusion of low-flow immunoglobulin over 4 days.

\section{Therapies}

All patients except in the isolated myocarditis (MY) group received aspirin (50 to $80 \mathrm{mg} / \mathrm{kg}$ day) and one high-dosage 
Table 2 Clinical signs differences depending on diagnosis

\begin{tabular}{|c|c|c|c|c|c|}
\hline & $\begin{array}{l}\text { Total } \\
(n=32)\end{array}$ & $\begin{array}{l}\mathrm{KD} \\
(n=11)\end{array}$ & $\mathrm{KS}(n=6)$ & $\mathrm{KM}(n=7)$ & $\mathrm{MY}(n=8)$ \\
\hline Age (years) & $5[1-18]$ & $2[0.6-7.5]$ & $5[1.7-6]$ & $8[7-8]$ & $8[0.9-17]$ \\
\hline Weight (kg) & $21[4.7-75]$ & $12[6-24]$ & $19[8.4-28]$ & $45[25.2-60]$ & $28[4.7-75]$ \\
\hline Height (cm) & $110[54-180]$ & $85[68-126]$ & $103[84-118]$ & $147[123-174]$ & $147[54-180]$ \\
\hline Sex ratio $(M / F)$ & $1.3(18 / 14)$ & $1.75(7 / 4)$ & $0.5(2 / 4)$ & $1.3(4 / 3)$ & $1.7(5 / 3)$ \\
\hline Fever (days) & $5.5[0-48]$ & $5[4-10]$ & $11[4-48]$ & $8[4-13]$ & $2[0-4]$ \\
\hline Principal features of KD & $2.75[0-5]$ & $4.2[2 *-5]$ & $4[4-5]$ & $3[3-3]$ & $0.4[0-2]$ \\
\hline Polymorphous exanthem & $19(59 \%)$ & $10(91 \%)$ & $6(100 \%)$ & $3(43 \%)$ & $1(13 \%)$ \\
\hline Changes in extremities & $15(47 \%)$ & $6(55 \%)$ & $4(67 \%)$ & $6(86 \%)$ & 0 \\
\hline $\begin{array}{l}\text { Bilateral bulbar } \\
\text { conjunctival injection } \\
\text { without exudate }\end{array}$ & $19(59 \%)$ & $10(91 \%)$ & $4(67 \%)$ & $6(86 \%)$ & 0 \\
\hline $\begin{array}{l}\text { Changes in lips and oral } \\
\text { cavity }\end{array}$ & $17(53 \%)$ & $9(82 \%)$ & $4(67 \%)$ & $4(57 \%)$ & $1(13 \%)$ \\
\hline $\begin{array}{l}\text { Cervical } \\
\text { lymphadenopathy } \\
(>1.5 \mathrm{~cm})\end{array}$ & $19(59 \%)$ & $11(100 \%)$ & $6(100 \%)$ & $2(29 \%)$ & $1(13 \%)$ \\
\hline Gastrointestinal symptoms & $18(56 \%)$ & $6(55 \%)$ & $1(17 \%)$ & $6(86 \%)$ & $4(50 \%)$ \\
\hline Respiratory distress & $6(19 \%)$ & 0 & $1(17 \%)$ & $3(43 \%)$ & $2(25 \%)$ \\
\hline Adenophlegmon & $3(9 \%)$ & 0 & 0 & $3(43 \%)$ & 0 \\
\hline Meningism & $2(6 \%)$ & 0 & 0 & $2(29 \%)$ & 0 \\
\hline
\end{tabular}

$K D$ complete Kawasaki disease, $K S$ Kawasaki syndrome, $M Y$ isolated myocarditis, $K M$ others (all COVID+) intravenous infusion $(2 \mathrm{~g} / \mathrm{kg})$ of immunoglobulins after a median time since first clinical sign of 5 [2-48] days. Eight patients $(33 \%)$ required a second infusion. Six received intravenous infusion of methylprednisolone $(2 \mathrm{mg} / \mathrm{kg}$ ) during 3 days. One KM patient with a myocarditis associated to COVID received anti-interleukin 1 therapy. One KS patient with polyarteritis nodosa was treated with cyclophosphamide. Twenty-four received broad-spectrum probabilistic antibiotic therapy. Finally, anticoagulation was used for teenagers and younger children presenting with a major inflammatory syndrome, hypoalbuminemia and/or prolonged decubitus. Amongst patients who received heparin, 4 had curative dosage and 7 had preventive dosage.

\section{Outcomes}

Two patients with myocarditis and one patient with Kawasaki syndrome died. One patient with myocarditis underwent emergency heart transplantation because of ventricular fibrillation storms 2 months after the initial episode. All the other

Fig. 2 Typical clinical feature of the COVID-19-linked myocarditis with Kawasaki clinical features
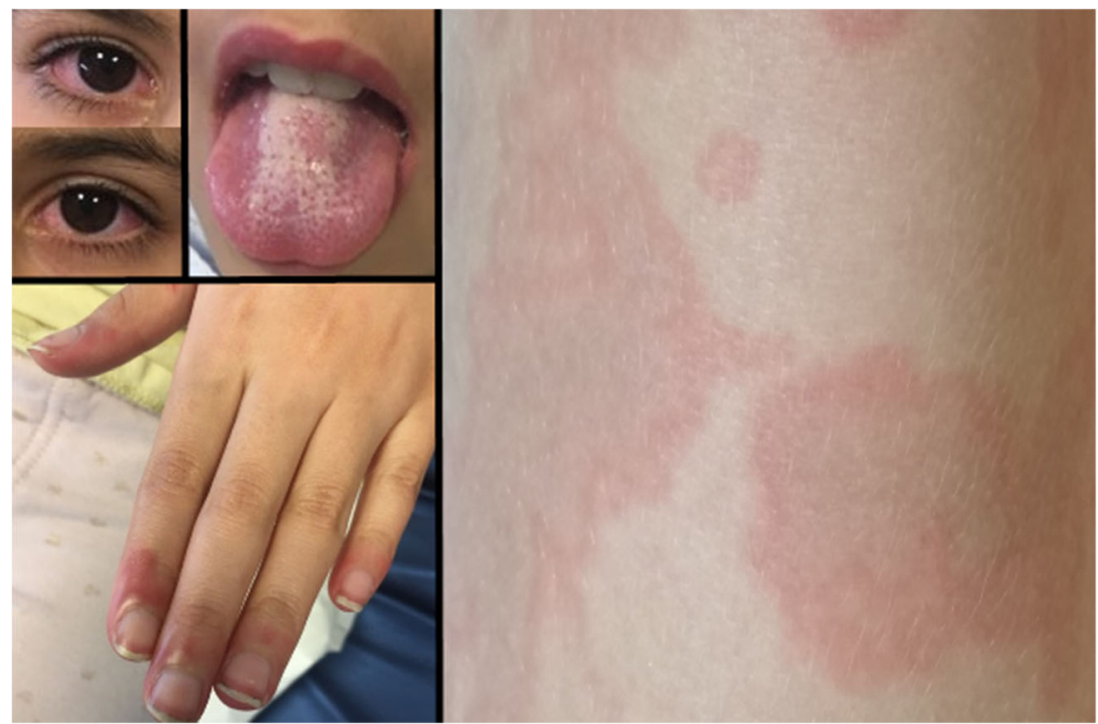
children who had a myocardial impairment recovered a normal myocardial function in 4.5 days [1-60]. One patient with Kawasaki syndrome presented with diffuse vasculitis, cerebral ischemia, acute limb ischemia and aortic dilation leading to severe aortic regurgitation requiring secondary surgery. The patient underwent successful aortic valve repair 1 year after the initial episode. The final diagnosis was polyarteritis nodosa. Two KD patients with complete Kawasaki disease kept a coronary dilatation.

Amongst the KM group (myocarditis associated with COVID-19), myocardial recovery was complete in all cases, as evidenced by cardiac resonance imaging performed in the month following the initial episode. Short- and mid-term outcomes of these children are presented in Table 3.

\section{Impact of the COVID-19 epidemic}

We report no increase in the incidence of complete Kawasaki disease after the 15th of February 2020 (Table 1). In our population, time delay between two occurrences of complete Kawasaki disease was unchanged before and after the onset of the COVID-19 pandemic $(p=0.25)$. Similarly, the incidence of isolated myocarditis remained stable before and after the start of the pandemic $(p=0.43)$. There were no cases of Kawasaki syndrome after February 2020, and no cases of KM children before February 2020.

\section{Discussion}

From our retrospective analysis, we described a new paediatric condition associating myocarditis, infection with SARSCoV-2 and multisystem inflammatory syndrome mimicking an incomplete Kawasaki disease. The clinical description of the children presenting this disease fills the gap between two well know conditions: isolated myocarditis and Kawasaki disease. As suggested by Calabri et al., this association is probably much more a new form of vasculitis, or COVID-19associated vasculitis, rather than a COVID-19-associated Kawasaki disease [10]. This emerging disease has indeed been named "SCiKH syndrome for SARS-CoV-2-induced Kawasaki-like hyperinflammatory syndrome" by Licciardi; or "MIS-C" for "multisystem inflammatory syndrome in children and adolescents" by the World Health Organization [11-14] or PIMS for paediatric multisystem inflammatory syndrome temporally associated with COVID-19 by the Royal College of Paediatrics in Child Health [15]. This profusion of new denominations sustains the idea of a new disease, which our results are consistent with.

Interestingly, our data showed that this new multisystem inflammatory disorder often exhibits digestive symptoms sometimes misinterpreted as an acute abdomen. It is an important fact to be known, given that it can mislead physicians towards surgery for suspected appendicitis with, in our case, a particularly complicated course. Also, predominant otorhinolaryngologic symptoms have been described: there were 2 cases of adenophlegmon in our cohort, as reported by Sandhaus [16].

In our study population, all the cases of combined myocarditis and incomplete Kawasaki syndrome occurred during the COVID-19 pandemic and had an exposure to SARS-CoV-2 and a biologically and/or radiologically confirmed COVID-19 diagnosis. Notably, some patients $(71 \%)$ had positive serologic tests for SARS-CoV-2 infection but negative PCR, suggesting that they probably had an asymptomatic COVID-19, as most children in previous cohort, and secondarily developed myocarditis/incomplete Kawasaki disease [17]. It was objectified that all but one had origins from the Mediterranean basin or from sub-Saharan Africa, which suggests an ethnic or genetic predisposition, as suggested by Moreira [18].

An increase in the incidence of Kawasaki syndromes and multisystem inflammatory response mimicking Kawasaki syndromes has been described in other centres where the epidemic of COVID-19 was particularly significant. Verdoni et al. reported a 30-fold increase in the incidence of Kawasaki syndrome in Bergamo (Italy) [19]. A similar comparison in our cohort shows an increase in incidence of 15 times. However, the incidence of Kawasaki disease did not increase during the pandemic in our centre. The increase in incomplete forms of Kawasaki syndromes in our population
Table 3 Outcome of KM group: 7 cases of myocarditis associated with COVID19

\begin{tabular}{llll}
\hline & Group KM initial & Group KM post-treatment* & $p$ \\
\hline Clinical & & & \\
$\quad$ Survivors, $n(\%)$ & & $7(100 \%)$ & \\
Biological & & & 0.002 \\
CRP (mg/L), $n[\min ; \max ]$ & $248[48 ; 354]$ & $17[0 ; 80]$ & 0.13 \\
Troponin $(\mathrm{ng} / \mathrm{L}), n[\min ; \max ]$ & $338[135 ; 7335]$ & $200[135 ; 346]$ & \\
Echocardiography & & & 0.11 \\
LV ejection fraction $(\%), n[\min ; \max ]$ & $50[30 ; 65]$ & $60[60 ; 65]$ & 0.07 \\
Mitral regurgitation, $n(\%)$ & $7(100 \%)$ & $4(57 \%)$ & \\
\hline
\end{tabular}


was only due to the occurrence of this new COVID-19 disease. However suspected, the physiological link between multisystem inflammatory response and COVID-19 has not been demonstrated yet [20-22]. As in the adult, it can be hypothesized that SARS-CoV-2 infection could induce an inappropriate immune response, causing an inflammatory reaction leading to vasculitis $[11,23,24]$. Another possibility is that inflammatory diseases like Kawasaki and myocarditis could directly result in an endothelial lesion provoked by a specific virus $[1,25,26]$. Finally, these types of inflammatory diseases could depend on the external viral pressure. In other terms, if the quantity of virus(es) circulating amongst the general population increases, children would tend to develop myocarditis and/or vasculitis, independently of the species of the virus.

In this cohort, therapy against myocarditis with incomplete Kawasaki syndrome consisted of an aggressive antiinflammatory treatment, comprising large doses of immunoglobulins and aspirin. It has been effective in this cohort, with a total regression of myocardial impairment. For this new form, the rationale for this treatment is well described by Ronconi et al. [27].

Importantly, all of the patients infected with SARS-CoV-2 had a positive outcome, with no mortality. Our results confirm the low morbi-mortality of COVID-19 in children [28], as noted also in France [29], with, to date, only one national case of mortality in children ages less than 14 years.

One of the challenges of such a study was the unavoidable small size of the samples possibly leading to interpretation biases. We noted a global increased incidence, of myocarditis/Kawasaki disease/Kawasaki syndrome during COVID-19 pandemic in our centre. Before the pandemic, some patients could be treated in peripheral hospitals of the region. This policy changed after the onset of the pandemic. As a result, at least 2 patients were sent to our centre while they would not have before the pandemic. The resulting bias in considering an increased incidence of cases is probably low but has to be noted on such a small cohort.

\section{Conclusion}

We retrospectively conducted a descriptive analysis of a monocentric cohort of children with inflammatory disease such as complete or incomplete Kawasaki disease and/or myocarditis. Incidences of Kawasaki disease and isolated myocarditis were stable over time before and after the onset of the pandemic. Our results showed that a new disease aroused after the onset of the COVID-19 pandemic combining myocarditis and clinical features of Kawasaki syndrome. This combination of myocarditis and multisystem inflammation clinically mimicking incomplete Kawasaki syndrome was associated with SARS-CoV-2 infection and had a better prognosis than isolated myocarditis. In children, COVID-19 presents with the features of a multi-organ inflammatory syndrome with multi-faceted clinical manifestations.

Authors' contributions JB, CO, ACG and LB: data collection

JB, LB and SP: data treatment

JB, LB and SP: manuscript writing

All authors: manuscript editing and approving

\section{Compliance with ethical statements}

Conflict of interest The authors declare that they have no conflict of interest.

Ethical approval This article does not contain any studies with human participants or animals performed by any of the authors.

Informed consent Informed consent was obtained from all individual whose photographies have been included in the manuscript.

\section{References}

1. Newburger JW, Takahashi M, Burns JC (2016) Kawasaki disease. J Am Coll Cardiol 67:1738-1749. https://doi.org/10.1016/j.jacc. 2015.12.073

2. Dietz SM, van Stijn D, Burgner D, Levin M, Kuipers IM, Hutten BA, Kuijpers TW (2017) Dissecting Kawasaki disease: a state-ofthe-art review. Eur J Pediatr 176:995-1009. https://doi.org/10. 1007/s00431-017-2937-5

3. Newburger JW, Takahashi M, Gerber MA, Gewitz MH, Tani LY, Burns JC, Shulman ST, Bolger AF, Ferrieri P, Baltimore RS, Wilson WR, Baddour LM, Levison ME, Pallasch TJ, Falace DA, Taubert KA (2004) Diagnosis, treatment, and long-term management of Kawasaki disease: a statement for health professionals from the committee on rheumatic fever, endocarditis, and Kawasaki disease, council on cardiovascular disease in the young, American Heart Association. PEDIATRICS 114:1708-1733. https://doi.org/ 10.1542/peds.2004-2182

4. Liguoro I, Pilotto C, Bonanni M, Ferrari ME, Pusiol A, Nocerino A, Vidal E, Cogo P (2020) SARS-COV-2 infection in children and newborns: a systematic review. Eur J Pediatr 179:1-18. https://doi. org/10.1007/s00431-020-03684-7

5. World Health Organisation https://www.covid19healthsystem.org/ countries/france/countrypage.aspx. Accessed 4 Jul 2020

6. Caforio ALP, Marcolongo R, Basso C, Iliceto S (2015) Clinical presentation and diagnosis of myocarditis. Heart 101:1332-1344. https://doi.org/10.1136/heartjnl-2014-306363

7. Zu ZY, Jiang MD, Xu PP, Chen W, Ni QQ, Lu GM, Zhang LJ (2020) Coronavirus disease 2019 (COVID-19): a perspective from China. Radiology 200490:E15-E25. https://doi.org/10.1148/radiol. 2020200490

8. Development Core Team R (2009) R: a language and environment for statistical computing. R Foundation for Statistical Computing, Vienna

9. Siegel RJ (1956) Nonparametric statistics for the behavioral sciences, international student edition. McGraw-Hill, New York Toronto London

10. Calabri GB, Formigari R (2020) Covid-19 and Kawasaki disease: a glimpse at the past for a predictable future. Pediatr Cardiol 41: 1075-1075. https://doi.org/10.1007/s00246-020-02385-0

11. Grimaud M, Starck J, Levy M, Marais C, Chareyre J, Khraiche D, Leruez-Ville M, Quartier P, Léger PL, Geslain G, Semaan N, Moulin F, Bendavid M, Jean S, Poncelet G, Renolleau S, Oualha 
M (2020) Acute myocarditis and multisystem inflammatory emerging disease following SARS-CoV-2 infection in critically ill children. Ann Intensive Care 10:69. https://doi.org/10.1186/s13613020-00690-8

12. Licciardi F, Pruccoli G, Denina M et al (2020) SARS-CoV-2induced Kawasaki-like hyperinflammatory syndrome: a novel COVID phenotype in children. Pediatrics:e20201711. https://doi. org/10.1542/peds.2020-1711

13. Hennon TR, Penque MD, Abdul-Aziz R, Alibrahim OS, McGreevy MB, Prout AJ, Schaefer BA, Ambrusko SJ, Pastore JV, Turkovich SJ, Gomez-Duarte OG, Hicar MD (2020) COVID-19 associated multisystem inflammatory syndrome in children (MIS-C) guidelines; a Western New York approach. Prog Pediatr Cardiol 57: 101232. https://doi.org/10.1016/j.ppedcard.2020.101232

14. Sperotto F, Friedman KG, Son MBF, VanderPluym CJ, Newburger JW, Dionne A (2020) Cardiac manifestations in SARS-CoV-2associated multisystem inflammatory syndrome in children: a comprehensive review and proposed clinical approach. Eur J Pediatr. https://doi.org/10.1007/s00431-020-03766-6

15. BPAIIG, North Thames Paediatric Network and South Thames Paediatric, Network (2020) Guidance - paediatric multisystem inflammatory syndrome temporally associated with COVID-19 (PIMS). In: RCPCH Royal College of Paediatrics and Child Health. https://www.rcpch.ac.uk/resources/guidance-paediatricmultisystem-inflammatory-syndrome-temporally-associatedcovid-19-pims. Accessed 5 Jul 2020

16. Sandhaus H, Crosby D, Sharma A, Gregory SR (2020) Association between COVID-19 and Kawasaki disease: vigilance required from otolaryngologists. Otolaryngol Head Neck Surg: 019459982093023. https://doi.org/10.1177/0194599820930238

17. Xu Y, Li X, Zhu B, Liang H, Fang C, Gong Y, Guo Q, Sun X, Zhao D, Shen J, Zhang H, Liu H, Xia H, Tang J, Zhang K, Gong S (2020) Characteristics of pediatric SARS-CoV-2 infection and potential evidence for persistent fecal viral shedding. Nat Med 26:502-505. https://doi.org/10.1038/s41591-020-0817-4

18. Moreira A (2020) Kawasaki disease linked to COVID-19 in children. Nat Rev Immunol 20:407-407. https://doi.org/10.1038/ s41577-020-0350-1

19. Verdoni L, Mazza A, Gervasoni A, Martelli L, Ruggeri M, Ciuffreda M, Bonanomi E, D'Antiga L (2020) An outbreak of severe Kawasaki-like disease at the Italian epicentre of the SARSCoV-2 epidemic: an observational cohort study. Lancet 395:17711778. https://doi.org/10.1016/S0140-6736(20)31103-X

20. Raba AA, Abobaker A (2020) Covid-19 and Kawasaki disease: an etiology or coincidental infection? Pediatr Infect Dis J 39:e213. https://doi.org/10.1097/INF.0000000000002779
21. Xu S, Chen M, Weng J (2020) COVID-19 and Kawasaki disease in children. Pharmacol Res 159:104951. https://doi.org/10.1016/j. phrs.2020.104951

22. Jones VG, Mills M, Suarez D, Hogan CA, Yeh D, Segal JB, Nguyen EL, Barsh GR, Maskatia S, Mathew R (2020) COVID19 and Kawasaki disease: novel virus and novel case. Hosp Pediatr 10:537-540. https://doi.org/10.1542/hpeds.2020-0123

23. Blanco-Melo D, Nilsson-Payant BE, Liu W-C et al (2020) Imbalanced host response to SARS-CoV-2 drives development of COVID-19. Cell 181:1036-1045.e9. https://doi.org/10.1016/j.cell. 2020.04.026

24. Koné-Paut I, Cimaz R (2020) Is it Kawasaki shock syndrome, Kawasaki-like disease or pediatric inflammatory multisystem disease? The importance of semantic in the era of COVID-19 pandemic. RMD Open 6:e001333. https://doi.org/10.1136/rmdopen-2020001333

25. Shirato K, Imada Y, Kawase M, Nakagaki K, Matsuyama S, Taguchi F (2014) Possible involvement of infection with human coronavirus 229E, but not NL63, in Kawasaki disease: Kawasaki disease and HCoV229E. J Med Virol 86:2146-2153. https://doi. org/10.1002/jmv.23950

26. Wang J, Li J, Ren Y, Shi H, Rong X, Zhang X, Shao Y, Wu R, Chu M, Qiu H (2020) Association between alanine aminotransferase/ aspartate aminotransferase ratio (AST/ALT ratio) and coronary artery injury in children with Kawasaki disease. Cardiol Res Pract 2020:1-7. https://doi.org/10.1155/2020/8743548

27. Ronconi G, Teté G, Kritas SK et al (2020) SARS-CoV-2, which induces COVID-19, causes kawasaki-like disease in children: role of pro-inflammatory and anti-inflammatory cytokines. J Biol Regul Homeost Agents 34. https://doi.org/10.23812/EDITORIALRONCONI-E-59

28. Sanna G, Serrau G, Bassareo PP, Neroni P, Fanos V, Marcialis MA (2020) Children's heart and COVID-19: up-to-date evidence in the form of a systematic review. Eur J Pediatr 179:1079-1087. https:// doi.org/10.1007/s00431-020-03699-0

29. Belot A, Antona D, Renolleau S, Javouhey E, Hentgen V, Angoulvant F, Delacourt C, Iriart X, Ovaert C, Bader-Meunier B, Kone-Paut I, Levy-Bruhl D (2020) SARS-CoV-2-related paediatric inflammatory multisystem syndrome, an epidemiological study, France, 1 March to 17 May 2020. Euro Surveill:25. https://doi. org/10.2807/1560-7917.ES.2020.25.22.2001010

Publisher's note Springer Nature remains neutral with regard to jurisdictional claims in published maps and institutional affiliations. 